\title{
Dual-tasking gait variability and cognition in late-life depression
}

\author{
Nicolette M. Gabel ${ }^{1}$, Natania A. Crane ${ }^{3}$, Erich T. Avery ${ }^{1}$, Rachel E. Kay ${ }^{1}$, Amanda Laurent ${ }^{1}$, Bruno Giordani ${ }^{1}$, \\ Neil B. Alexander ${ }^{1,2}$ and Sara L. Weisenbach ${ }^{1,3,4}$ \\ ${ }^{1}$ University of Michigan, Ann Arbor, MI, USA \\ ${ }^{2}$ VA Ann Arbor Healthcare System, Ann Arbor, MI, USA \\ ${ }^{3}$ University of Illinois at Chicago, Chicago, IL, USA \\ ${ }^{4}$ Jesse Brown VA Medical Center, Chicago, IL, USA \\ Correspondence to: S. L. Weisenbach, PhD, E-mail: sweisenbach@psych.uic.edu
}

Objectives: Studies have demonstrated an association between major depressive disorder (MDD) symptoms and fall risk in older adults, which may be at least partially mediated by executive functioning skills. There have also been observations of increased gait variability associated with fall risk and disease. This preliminary study first sought to understand whether gait variability in the context of dual task cost differs among older adults with MDD, relative to those with no history of psychiatric illness, and second, to identify relationships between gait variability measures and cognitive functioning in the context of MDD.

Methods: We recruited 15 older adults with MDD and 17 non-depressed (ND) community-dwelling older adults. All participants had impaired balance based on unipedal stance time. Assessments included neuropsychological measures and measures of gait variability using an instrumented gait mat (GAITRite ${ }^{\odot}$ ) in the context of dual task relative to single task performance (i.e., dual task cost).

Results: The groups did not differ on any gait variability parameters. The MDD group demonstrated poorer performance in the psychomotor speed domain, relative to the ND group, but cognitive functioning between the groups in other domains was equivalent. In MDD, increased variability in stride time, stride velocity, and swing time during dual-tasking were associated with poorer executive functioning and visual memory. In ND, no significant relationships between gait variables and cognitive performance were observed.

Conclusions: Findings suggest that unique cognitive mechanisms underlie mobility problems associated with fall risk in late-life depression. Copyright (C) 2015 John Wiley \& Sons, Ltd.

Key words: late-life depression; gait; executive functioning; balance; fall; mobility

History: Received 28 February 2015; Revised 12 July 2015; Accepted 13 July 2015; Published online 6 August 2015 in Wiley Online Library (wileyonlinelibrary.com)

DOI: 10.1002 /gps.4340

\section{Introduction}

Increased variability in gait-such as stride-to-stride fluctuations in length of stride ("stride length") and time between steps ("swing time")—predicts falls in older adults (Hausdorff et al., 2001), while increased variability in velocity ("stride velocity") has been associated with neurodegenerative processes such as Alzheimer's disease (Nakamura et al., 1996). In otherwise healthy older adults, history of falls has been associated with more unsteadiness while walking, with less consistency and overall poorer gait regulation (Hausdorff et al., 1997).

Studies of cognitive factors associated with fall risk have focused on executive functioning (EF), which encompasses cognitive abilities used in the efficient manipulation of information and purposeful behavior (Lezak et al., 2013). EF moderates the relationship between physiological function and falls (Rapport et al., 1998; Herman et al., 2010), likely due to the necessity 
of adequate EF in appropriately regulating and adjusting movement to limitations and obstacles (Wright et al., 2011). Poorer EF has been associated with reduced performance on numerous gait measures, including those indicative of balance control while walking and gait speed (Martin et al., 2013), as well as increased gait variability (Sheridan et al., 2003), all of which pose an increased risk for falling. Furthermore, reduced EF has been found to uniquely predict falls in medical inpatients (Rapport et al., 1998) and community-dwelling older adults (Herman, et al., 2005). In their review of the role of EF in gait, Yogev and colleagues (2008) describe gait as an "attention-demanding, high-level, controlled task" that is vulnerable to the burden of additional attentional demands, such as the performance of concurrent cognitive or motor tasks (i.e., dual-tasking; DT). Growing evidence connecting DT with performance on traditional tests of EF suggest that the former may be considered a functional analogue of the latter (Herman et al., 2005; Wright et al., 2011). DT has been shown to increase gait variability (specifically in the time between footfalls of the same foot, i.e., "swing time") in older adults at risk for falls, and these older adults performed worse on EF tests than older adults with no history of falls (Springer et al., 2006).

Depression in late life has been associated with cognitive dysfunction, including deficits in processing speed, memory, and EF (Lesser et al., 1996; Lockwood et al., 2002; Baudic et al., 2004; Butters et al., 2004). In late-life depression, the development of concurrent executive dysfunction and depressive symptoms may reflect a shared underlying etiology, such as frontostriatal pathology (Alexopoulos et al., 2002). Depression is an independent risk factor for falls in older adults, with characteristic cognitive deficits likely contributing to changes in motor coordination and attentional abilities (Iaboni and Flint, 2013). A pilot study by Wright and colleagues (2011) compared community-dwelling older adults with major depressive disorder (MDD) to non-depressed (ND) controls on traditional EF measures as well as on a novel measure of timed stepping accuracy (i.e., the Walking Trail-Making Test; W-TMT). Participants with MDD performed W-TMT-B more slowly than did ND controls and demonstrated a higher DT cost (i.e., DTSingle Task/Single Task; "DTC"). Additionally, WTMT-B performance was significantly correlated with traditional measures of EF.

Past research suggests, but has not strongly determined, that gait variability in the context of DT differs among older adults with MDD, relative to those with no history of psychiatric illness; however, to date, most studies investigating this relationship have measured depression with self-report inventories of depressive symptoms, and it is unclear how many individuals in those studies met criteria for MDD. Relationships between gait variability as a function of DT cost and cognitive functioning (including EF) likely differ between older adults with and without MDD. In the present study, we hypothesized that (1) older adults with MDD would demonstrate more variability on DT gait measures relative to single task (ST) gait measures and poorer performance on cognitive measures; (2) greater gait variability during DT (relative to ST) would be associated with poorer performance on cognitive measures, specifically EF (Holtzer et al., 2014); and (3) relationships among gait variability and EF would be stronger in the MDD group, reflecting primary effects of depression on cognition and gait, as well as possible secondary effects of depression on gait (i.e., via cognitive mechanisms).

\section{Methods}

\section{Participants}

Participants were 32 women and men ages 61 to 88 years $[M=73.03$, standard deviation $(S D)=7.98]$ who were recruited from community advertisements, university research participant registries, and outpatient clinics at University of Michigan. Of these participants, 15 had a diagnosis of MDD (nine women and six men; age $M=70.73, S D=7.84)$ as determined using the Structured Clinical Interview for DSM-IV (Spitzer et al., 1994), and 17 had no history of MDD or any other psychiatric disorders (nine women and eight men; age $M=75.06, S D=7.76$ ). See Table 1 for further demographic and clinical information.

Exclusion criteria included diagnoses of bipolar depression or psychosis; pre-existing dementia diagnosis; traumatic brain injury with loss of consciousness $>3$ min; movement disorders or changes in gait associated with parkinsonism, cerebrovascular accident, epilepsy, or amputation of a lower extremity; current substance abuse; medical instability (e.g., acute, terminal, or worsening of major medical condition); current major medical treatment (e.g., radiation and chemotherapy); inability to ambulate without an assistive device; severe weight-bearing pain that would interfere with gait performance; and inability to speak fluent English.

Participants were included in the study only if they obtained scores above 24 (out of 30) on the Minimental status exam (Folstein et al., 1975) to rule out 
Table 1 Mean and standard deviation of demographic variables by group

\begin{tabular}{|c|c|c|}
\hline & $\begin{array}{c}\text { ND } \\
(n=17) \\
\text { Mean (SD) }\end{array}$ & $\begin{array}{c}\text { MDD } \\
(n=15) \\
\text { Mean (SD) }\end{array}$ \\
\hline Age & $75(7.76)$ & $71(7.84)$ \\
\hline $\begin{array}{l}\text { Charlson comorbidity index } \\
\text { (age-corrected; } \\
\text { Charlson et al., 1987) }\end{array}$ & $4.33(1.50)$ & $3.47(1.25)$ \\
\hline Education (years) & $16(2.26)$ & $16(2.26)$ \\
\hline Hamilton Depression Rating Scale ${ }^{a}$ & $2.2(1.70)$ & $17.5(5.73)$ \\
\hline Taking antidepressant(s) $(\mathrm{Y} / \mathrm{N})^{\mathrm{b}}$ & N/A & $10 / 5$ \\
\hline Age of depression onset ${ }^{\mathrm{C}}$ & $\mathrm{N} / \mathrm{A}$ & $34.6(20.2)$ \\
\hline
\end{tabular}

Note.

${ }^{\mathrm{a}} t(15.12)=-9.6, p<0.01$.

${ }^{b}$ Two were treated with duloxetine only, two were treated with citalopram only, one was treated with mirtazapine only, one was treated with citalopram and bupropion, one was treated with citalopram and nortriptyline, one was treated with citalopram and trazodone, one was treated with fluoxetine and bupropion, and one was treated with duloxetine, trazodone, and bupropion.

${ }^{c} n=12$, range $10-73, n=9<$ age 50 years; $n=30 \geq$ age 50 years.

$\mathrm{ND}$, non-depressed; MDD, major depressive disorder; SD, standard deviation.

generalized cognitive impairment and demonstrated fall risk as measured by a unipedal stance time (UST; i.e., the duration of time a participant was able to stand on one foot with eyes open and free movement of arms) less than $5 \mathrm{~s}$ (normal range UST is $30-45 \mathrm{~s}$; Vellas et al., 1997). High risk of falls was part of the inclusion criteria in order to examine gait and cognitive variables, and the relationships between gait and cognition, among people for whom such relationships are likely to be most relevant.

\section{Measures}

Neuropsychological Measures. Verbal phonemic fluency was measured using the Controlled Oral Word Association Test (Benton et al., 1994), and semantic fluency was measured using animal naming (Benton et al., 1994). For both tests, the total number of acceptable words served as the score. Trail-Making Test A and B (Reitan and Reitan, 1985) was administered to assess motor speed, visual scanning, sequencing, and attentional shifting abilities. Time to completion served as the score for Trail A and was inverted for ease of interpretation (i.e., less time $=$ better performance). Trail $B$ requires an additional attentional shifting component, and the ratio of time to completion (inverted) for Trail $\mathrm{B}$ to Trail A was derived to provide a measurement of this set-shifting component while controlling for psychomotor speed. Verbal learning, recall, and recognition were measured using the California Verbal Learning Test-Second Edition (Delis et al., 2000), a list-learning task of 16 words over five trials followed by short (1-3 min) and long delay (18$20 \mathrm{~min}$ ) recall trials. A yes-no recognition trial was then administered. Scoring was completed according to the manual. Visual learning, recall, and recognition were assessed using the Brief Visual Memory Test-Revised (Benedict, 1997), which includes six stimulus figures presented to participants for $10 \mathrm{~s}$, repeated for three learning trials, followed by delayed recall $(25 \mathrm{~min})$ and recognition trials. Scoring was completed according to the manual. Manual speed and dexterity were assessed with the Purdue Pegboard Test (Strauss et al., 2006). Number of pegs placed in $30 \mathrm{~s}$ for the right hand, left hand, and both hands served as performance measures.

Parametric go/no-go stop task. The parametric go/ no-go stop (PGNGS) task measures sustained attention (go hits) and set-shifting as the task becomes more difficult; processing speed (go response time), including simple and subsequently more challenging conditions of responding with no-go and stop rules; and inhibitory control, including the ability to stop an unwanted, prepotent response (correct rejections) and/or the failure to do so (commissions). The PGNGS task consists of two separate levels, each with three conditions, which were completed in order of ascending difficulty, and are based upon contextual inhibition, wherein the target and lure sets change depending upon the context (e.g., previous response). Table 2 illustrates the conditions in sequential order,

Table 2 PGNGS task conditions in sequential order

\begin{tabular}{|c|c|c|}
\hline Condition & Targets & Rule \\
\hline \multicolumn{3}{|l|}{ Level 1} \\
\hline 2 Target go & 2 (R and S) & $\begin{array}{l}\text { Respond to all "R" and } \\
\text { "S" stimuli }\end{array}$ \\
\hline 2 Target no-go & 2 (R and S) & $\begin{array}{l}\text { Non-repeating, respond } \\
\text { to "R" and "S" in alternation }\end{array}$ \\
\hline 2 Target stop & 2 (R and S) & $\begin{array}{l}\text { Stop-signal, respond to "R" } \\
\text { and "S" unless interrupted } \\
\text { by a stop sign }\end{array}$ \\
\hline \multicolumn{3}{|l|}{ Level 2} \\
\hline 3 Target go & $3(\mathrm{R}, \mathrm{S}$, and $\mathrm{T})$ & $\begin{array}{l}\text { Respond to all "R," "S," } \\
\text { and "T" stimuli, regardless } \\
\text { of order }\end{array}$ \\
\hline 3 Target no-go & $3(\mathrm{R}, \mathrm{S}$, and $\mathrm{T})$ & $\begin{array}{l}\text { Non-repeating, respond to } \\
\text { "R," "S," and "T," not twice } \\
\text { in a row }\end{array}$ \\
\hline 3 Target stop & $3(\mathrm{R}, \mathrm{S}$, and $\mathrm{T})$ & $\begin{array}{l}\text { Stop-signal, respond to "R," } \\
\text { "S," and "T" unless } \\
\text { interrupted by a stop sign }\end{array}$ \\
\hline
\end{tabular}


by level (number of targets). Accuracy scores for each set of trials (i.e., go trials, no-go trials, and stop trials) were derived by dividing the number of correct hits by the sum of correct hits and errors of commission (i.e., hits/hits + errors).

Gait variables. A GAITRite Portable Walkway System ${ }^{\circledR}$ was used to collect and analyze gait variables. This portable $16 \mathrm{ft}$ long computerized mat is composed of embedded pressure sensors, which collect gait information while the participant walks across it wearing their own shoes. Variables of interest collected from the GAITRite $^{\circledR}$ mat include stride time (i.e., time elapsed in seconds between two consecutive footfalls of the same foot; associated with gait pace); stride velocity (ratio of stride length in centimeters/stride time; associated with gait pace); and swing time (time in seconds between the last contact with the mat and the next footfall of the same foot; associated with gait rhythm; Lord et al., 2013).

\section{Procedure}

After completing an initial telephone screen, all participants signed written informed consent prior to participation, as approved by the University of Michigan and VA Ann Arbor Healthcare System Institutional Review Boards. A licensed psychologist (S.L.W.) or trainee under her supervision then administered a clinical interview including the Hamilton Depression Rating Scale (Hamilton, 1960) and Structured Clinical Interview for DSM-IV (Spitzer et al., 1994), as well as the Mini-mental status exam (Folstein et al., 1975) and UST (Vellas et al., 1997). Participants who met inclusion criteria were then administered neuropsychologi$\mathrm{cal}$ and gait assessments on separate days.

A narrowed walkway was created on the GAITRite $\left.^{(}\right)$mat specifically for this study, in order to make the task more challenging (Brown et al., 2002), consisting of two blue lines of tape spaced $25 \mathrm{~cm}$ apart. Participants were instructed to stay between the lines while performing the tasks. Height, weight, date of birth, shoe size, sex, and leg length (measuring from anterior superior iliac spine to lateral malleolus) were entered into the GAITRite ${ }^{\complement}$ computer prior to beginning walkway trials.

The battery consisted of three trials each of an ST (i.e., walk the length of the mat at normal walking pace, turn around, and return to starting point) and DT (i.e., walk the same length of the mat while reciting alternating letters (e.g., a, c, e...) starting with either a or b; or counting backwards by $2 \mathrm{~s}$, starting at either 95 or 94). All participants completed the ST trials first. During DT trials, the participant was asked to pay special attention to correctly perform the cognitive task, even at the expense of normal walking speed. The total number of correct responses and errors during DT was collected. A trial began when the participant first stepped onto the mat, was paused briefly when they reached the end of the mat and turned around, and began again with their first step onto the mat at return. Timing stopped when their last foot left the mat. Processing of GAITRite ${ }^{\mathbb{C}}$ data was completed by trained research assistants following a structured protocol using GAITRite ${ }^{\circledR}$ processing software version P4. Data were consolidated and cleaned via the "GAITRite ${ }^{\complement}$ Editor" and "Advanced Foot Separation" to identify separate footsteps and footfall pattern.

\section{Statistical analyses}

Neuropsychological test scores were combined into domains based on theoretically derived groupings. Scores on all tests included in domains were standardized to $z$-scores, using the entire group's scores, to aid analysis and interpretation. Specifically, Processing speed was composed of Trail A and the reaction time scores for the PGNGS test (i.e., Go trial reaction time and no-go reaction time). Internal consistency was improved by excluding stop trial reaction time and was acceptable $(\alpha=0.715)$. Verbal memory was composed of California Verbal Learning Test short delay free, short delay cued, long delay free, and long delay cued recall, and recognition accuracy. This scale demonstrated excellent internal consistency $(\alpha=0.96)$. Visual Memory was composed of scores on the Brief Visual Memory subtests total recall, delayed recall, and recognition. Internal consistency was acceptable $(\alpha=0.74)$. Psychomotor speed was composed of Purdue Pegboard number of pegs placed in $30 \mathrm{~s}$ for the right, left, and both hands. Internal consistency for this scale was good $(\alpha=0.85)$. Because of poor internal consistency among measures (i.e., $\alpha=\mathbf{0 . 0 1}$ ), an executive functioning domain was not utilized. Trails ratio, Controlled Oral Word Association Test, animal naming, and PGNGS accuracy for six trial types were analyzed independently, which were theoretically consistent with the proposition that EF encompasses several non-uniform cognitive functions (Lezak et al., 2013), and have been demonstrated to be separable and to differentially contribute to performance of higher level cognitive tasks when subjected to factor analysis (Miyake et al., 2000).

Coefficient of variance $(\mathrm{CV})$ scores for stride time, swing, time, and stride velocity, respectively, were derived to represent variability in gait performance during 
DT and ST trials by dividing the SD by the mean and multiplying by 100 [i.e., $C V=S D /(M \times 100)$ ]. ST $C V$ scores for each gait variable were subtracted from the DT CV score and divided by the ST CV score (i.e., $\mathrm{DT}-\mathrm{ST} / \mathrm{ST}$ ) to create measures of gait variability in the context of DT cost (i.e., DTC-CV).

Analyses of variance were performed to compare neuropsychological performance between MDD and ND groups. Because of non-normal distributions of gait variability measures, Mann-Whitney $U$ tests of independent samples were used to test for significant group differences in these variables. Estimated effect sizes for Mann-Whitney $U$ tests were calculated using the following formula: $r=z / \sqrt{ } n$ (Field, 2009). Spearman's Rho correlations were then used to explicate the relationships among the gait and neuropsychological performance for the entire sample and for each group separately.

\section{Results}

Descriptive statistics, significant analysis of variance results, and significant Mann-Whitney $U$ results for gait variables and neuropsychological test scores are provided in Table 3.

Mann-Whitney $U$ tests demonstrated no significant group differences in gait variability measures (i.e., DTC$\mathrm{CV}$; Table 3). The MDD group made fewer correct responses during the DT condition, relative to the ND group, but had a similar number of errors. When neuropsychological performance was compared by group, the MDD group demonstrated significantly slower performance in the psychomotor speed domain (median $=-0.55$, $S D=0.81)$ compared with the ND group (median $=0.07$, $S D=0.85 ; U(1,30)=44.5, p=0.04$; Table 4$)$. No other significant differences were found.
In the sample as a whole, a significant negative correlation was demonstrated between DTC-CV stride time and animal naming $\left(r_{s}=-0.41, p=0.03\right)$ and between DTC-CV stride time and 2 Target No-Go Accuracy $\left(r_{s}=-0.54, p=0.00\right)$. DTC-CV swing time was negatively correlated with 2 Target No-Go Accuracy $\left(r_{s}=-0.47\right.$, $p=0.01$ ), and DTC-CV stride velocity was negatively correlated with 3 Target Stop Accuracy $\left(r_{s}=-0.43\right.$, $p=0.03$; Supporting Information Table S1).

When the sample was split by depression status, the following significant relationships were found (Tables 5 and 6). In the MDD group, DTC-CV stride time was negatively correlated with animal naming $\left(r_{s}=-0.58, \quad p=0.04\right), \quad 3$ Target Go Accuracy $\left(r_{s}=-0.68, p=0.01\right)$, and 2 Target No-Go Accuracy $\left(r_{s}=-0.86, p=0.00\right)$. DTC-CV swing time was negatively correlated with Trails Ratio $\left(r_{s}=-0.53, p=0.04\right)$ and 2 Target No-Go Accuracy $\left(r_{s}=-0.77, p=0.00\right)$. DTC-CV stride velocity was negatively correlated with Visual Memory $\left(r_{s}=-0.74, p=0.00\right)$. In the ND group, there were no significant relationships between gait variability measures and cognitive performance.

\section{Discussion}

Findings suggest that among older patients with MDD at risk of falling, poorer cognitive performance (in the domains of visual memory and EF) is predictive of sub-optimal performance on measures of gait variability in the context of DT relative to single tasking (i.e., swing time, Stride Time, and stride velocity) that have been linked to fall risk (Hausdorff et al., 2001) and disease (Blin et al., 1990; Nakamura et al., 1996). In contrast, cognitive abilities do not appear to be predictive of gait variability in the context of DT in ND elders, also at risk of falling. This preliminary study is the first

Table 3 Means and standard deviations for gait variables of interest, by group

\begin{tabular}{|c|c|c|c|c|c|c|}
\hline & $\begin{array}{l}\text { Whole sample } \\
\qquad(n=32) \\
\text { Mean (SD) }\end{array}$ & $\begin{array}{c}\text { ND } \\
(n=17) \\
\text { Mean (SD) }\end{array}$ & $\begin{array}{c}\text { MDD } \\
(n=15) \\
\text { Mean (SD) }\end{array}$ & $U$ & $p$ & $r$ \\
\hline 1. ST stride time (s) & $1.2(0.1)$ & $1.1(0.1)$ & $1.2(0.1)$ & 156 & 0.30 & 0.19 \\
\hline 2. DT stride time (s) & $1.4(0.2)$ & $1.5(0.3)$ & $1.3(0.2)$ & 89 & 0.15 & -0.26 \\
\hline 3. DTC-CV stride time & $1.2(1.3)$ & $1.6(1.6)$ & $0.7(0.8)$ & 87 & 0.13 & -0.27 \\
\hline 4. ST swing time (s) & $0.4(0.0)$ & $0.4(0.0)$ & $0.4(0.0)$ & 140 & 0.66 & 0.08 \\
\hline 5. DT swing time (s) & $0.4(0.1)$ & $0.5(0.1)$ & $0.4(0.1)$ & 88 & 0.14 & -0.26 \\
\hline 6. DTC-CV swing time & $0.7(1.0)$ & $1.1(1.2)$ & $0.3(0.4)$ & 79 & 0.07 & -0.32 \\
\hline 7. ST stride velocity $(\mathrm{cm} / \mathrm{s})$ & $103.9(23.1)$ & $107.6(28.1)$ & $99.8(15.6)$ & 109 & 0.50 & -0.12 \\
\hline 8. DT stride velocity $(\mathrm{cm} / \mathrm{s})$ & $82.4(19.3)$ & 79.4 (21.9) & $85.7(16.1)$ & 149 & 0.43 & 0.14 \\
\hline 9. DTC-CV stride velocity & $0.8(1.1)$ & $1.1(1.2)$ & $0.5(0.8)$ & 85 & 0.11 & -0.28 \\
\hline 10. DT correct responses & $9.2(3.5)$ & $10.6(3.9)$ & $7.7(2.3)$ & 72.5 & $0.04^{*}$ & -0.37 \\
\hline 11. DT response errors & $1.1(1.3)$ & $1.3(1.5)$ & $0.8(0.2)$ & 100 & 0.31 & 0.19 \\
\hline
\end{tabular}

Note: ST, single task; DT, dual task; DTC-CV, dual task cost coefficient of variability; ND, non-depressed; MDD, mild depressive disorder; SD, standard deviation. 
Table 4 Means and standard deviations for cognitive variables of interest, by group

\begin{tabular}{|c|c|c|c|c|c|c|}
\hline & $\begin{array}{l}\text { Whole sample } \\
\quad(n=32) \\
\text { Mean (SD) }\end{array}$ & $\begin{array}{c}\text { ND } \\
(n=17) \\
\text { Mean (SD) }\end{array}$ & $\begin{array}{c}\text { MDD } \\
(n=15) \\
\text { Mean (SD) }\end{array}$ & $U$ & $p$ & $r$ \\
\hline 1. Animal naming (raw score) & $18.7(4.5)^{\mathrm{a}}$ & $17.9(3.8)^{\mathrm{e}}$ & $19.7(5.1)^{\mathrm{h}}$ & 117 & 0.40 & 0.09 \\
\hline 2. COWA (raw score) & $40.8(10.1)$ & $37.9(9.0)$ & $44.0(10.5)$ & 177 & 0.06 & 0.33 \\
\hline 3. Trails ratio (s) & $-2.4(1.0)^{b}$ & $-2.6(1.2)^{f}$ & $-2.3(0.6)$ & 124.5 & 0.86 & 0.03 \\
\hline 4. $2 \mathrm{~T}$ go accuracy & $0.97(0.05)^{\mathrm{a}}$ & $0.97(0.04)^{\mathrm{g}}$ & $0.96(0.06)^{\mathrm{g}}$ & 105 & 0.77 & 0.07 \\
\hline 5. 3 T go accuracy & $0.95(0.08)^{\mathrm{a}}$ & $0.96(0.07)^{\mathrm{g}}$ & $0.95(0.08)^{g}$ & 74.5 & 0.29 & -0.23 \\
\hline 6. 2 T no-go accuracy & $0.88(0.16)^{a}$ & $0.89(0.15)^{g}$ & $0.87(0.16)^{9}$ & 94 & 0.87 & -0.04 \\
\hline 7. 3 T no-go accuracy & $0.77(0.18)^{\mathrm{c}}$ & $0.78(0.13)^{\mathrm{h}}$ & $0.75(0.23)^{i}$ & 84 & 0.77 & 0.07 \\
\hline 8. 2 T stop accuracy & $0.85(0.18)^{a}$ & $0.88(0.20)^{\mathrm{g}}$ & $0.82(0.15)^{9}$ & 60.5 & 0.09 & -0.33 \\
\hline 9. 3 T stop accuracy & $0.91(0.09)^{\mathrm{C}}$ & $0.89(0.11)^{\mathrm{h}}$ & $0.92(0.07)^{i}$ & 81 & 0.87 & 0.03 \\
\hline 10. Processing speed (z-score) & $0.0(1.0)$ & $0.0(0.9)$ & $-0.1(1.0)$ & 123 & 0.88 & 0.03 \\
\hline 11. Verbal memory (z-score) & $0.0(1.0)$ & $0.0(0.6)$ & $0.0(1.2)$ & 129 & 0.97 & 0.01 \\
\hline 12. Visual memory ( $z$-score) & $0.0(1.0)^{\mathrm{a}}$ & $0.0(0.7)^{\mathrm{e}}$ & $0.0(1.0)^{h}$ & 103 & 0.82 & 0.05 \\
\hline 13. Psychomotor speed (z-score) & $0.0(1.0)^{d}$ & $0.3(0.8)^{9}$ & $-0.3(0.8)^{i}$ & 44.5 & $0.04^{\star}$ & 0.40 \\
\hline
\end{tabular}

Note: ND, non-depressed; MDD, mild depressive disorder; PGNGS, parametric go/no-go stop; SD, standard deviation; COWA, Controlled Oral Word Association Test; 2 T, PGNGS 2 target; 3 T, PGNGS 3 target. ${ }^{*}$ Mean difference significant at $p<0.05$.

${ }^{\mathrm{a}} n=28,{ }^{\mathrm{b}} n=31,{ }^{\mathrm{c}} n=25,{ }^{\mathrm{d}} n=26,{ }^{\mathrm{e}} n=15,{ }^{\mathrm{f}} n=16,{ }^{\mathrm{g}} n=14,{ }^{\mathrm{h}} n=13,{ }^{\mathrm{i}} n=12$,

known study to investigate relationships between gait variability and cognitive performance among older patients with MDD, and it strengthens what is known about the commonly observed triad of gait disturbance, depressive symptoms, and cognitive problems in geriatric patients (Hajjar et al., 2009). Importantly, it suggests that mechanisms of gait problems may be different in depressed, relative to ND elders, with cognitive processes contributing to a greater degree in the former than the latter.

There is a great deal of literature documenting executive dysfunction and other cognitive difficulties in older patients with depression, and it is widely believed that a common mechanism (i.e., disruption to frontostriatal-limbic circuitry) may contribute to both classes of symptoms (Alexopoulos et al., 2002). More recently, the literature has broadened to include gait disturbance as a third symptom class that may also have shared etiology (Hajjar et al., 2009; Hsu et al., 2014; Taylor et al., 2014). The MDD group demonstrated slower performances on tests of psychomotor speed compared with the ND group, which is consistent with previous literature (Lockwood et al., 2002) and the inclusion of psychomotor retardation symptoms in the diagnostic criteria for depression (American Psychiatric Association, 2014). It was surprising that the MDD and ND groups did not differ on other cognitive measures, particularly measures of EF, given the ever-growing literature supporting this association (Butters et al., 2004). It is notable that both the MDD and ND participants in this sample were highly educated, and thus likely have an elevated level of cognitive reserve (Lenehan et al., 2015). Cognitive difficulties in the context of depression may be most evident in samples with a broader range of premorbid cognitive abilities.

Contrary to expectation, no differences in performance were observed between groups on parameters of gait variability measured under DT relative to ST conditions. The sample studied consisted entirely of people at risk for falls, based on UST $<5 \mathrm{~s}$ (Vellas et al., 1997), which suggests that both groups exhibit gait dysfunction, and the effect size for depression status on gait variability may therefore be small. While the small sample size of this preliminary study limits our ability to demonstrate differences in gait variability between the groups, it is also possible that MDD does not directly affect gait variability during DT, compared with the well-demonstrated impact of other neuropsychological syndromes, such as Parkinson's disease (Blin et al., 1990). Rather the mechanisms underlying sub-optimal gait performance may be most relevant when distinguishing between those with and without depression at risk of falls. It is especially notable that the MDD group gave fewer correct responses during DT conditions, relative to the ND group, suggesting greater interference on cognitive processes during DT, in the context of being given explicit instructions to pay special attention to the cognitive task.

There were a few limitations to consider in making conclusions about the implications of this study. First, this is a small sample intended as a preliminary study. Thus, findings are intended to pave the way for more directed investigations of mechanisms underlying the relationship between depression and fall risk in larger samples. Given that the focus of this study was on cognition and gait variability, there may be other factors 







(such as balance deficits) that are more prominent contributors to fall risk and may exacerbate fall risk in ND older adults in particular. Future studies may therefore profit from inclusion of additional variables, such as level daily activity level, and measures specific to the mechanisms of balance itself. Second, two-thirds of our depressed participants were using antidepressants at the time of the study, which have been demonstrated to increase fall risk (Hartikainen et al., 2007), and may have impacted the associations among cognitive and gait variables. Again, a larger sample would allow for analysis of the potential contribution of antidepressant use to these relationships. Third, as mentioned previously, this is a highly educated, primarily Caucasian sample, and results may not generalize to more heterogeneous or less educated samples.

\section{Conclusions}

Our findings implicate cognition as a potential target for interventions to address fall risk in older adults with late-life depression. The effectiveness of problemsolving therapy has been demonstrated in depressed older adults with executive function impairments to reduce disability and depression symptoms (Alexopoulos et al., 2003), although further research is needed to measure the potential impact of this intervention (or similar ones) on functional abilities related specifically to gait. Indeed, multifactorial approaches appear to be the most appropriate and effective means of preventing falls in atrisk older adults (Chang et al., 2004), and our results suggest that future research and interventions for fall risk (in LLD in particular) should incorporate cognitive functioning as a target for assessment and treatment

\section{Conflict of interest}

No author declares any conflict of interest.

\section{Key point}

- Variability of stride time, stride velocity, and swing time during DT (relative to single tasking) were similar in depressed and never-depressed older adults with balance impairment, but measures of $\mathrm{EF}$ and visual memory were uniquely associated with gait variability parameters in the context of DT in the depressed group only. Findings suggest that unique cognitive mechanisms underlie mobility problems associated with fall risk in latelife depression.

\section{Acknowledgements}

We would like to thank Ciaran Considine for his assistance with data collection. Neuropsychological data have been included for nine participants in Wright et al. (2011).

This work was supported by VA Level One Career Development Award (S. L. W), Jack L. Berman, MD., and Barbara A. Berman, PhD. Depression Research Fund Award (S. L. W), Michigan Institute for Clinical and Health Research Pilot Grant Fund, and University of Michigan Claude D. Pepper Older Americans Independence Centers Trainee Support Award, grant number 5 P30 AG024824 (S. L. W).

\section{References}

Alexopoulos GS, Kiosses DN, Klimstra S, Kalayam B, Bruce ML. 2002. Clinical presentation of the "depression-executive dysfunction syndrome" of late life. Am J Geriatr Psychiatry 10: 98-106.

Alexopoulos GS, Raue P, Arean P. 2003. Problem-solving therapy versus supportive therapy in geriatric major depression with executive dysfunction. Am J Geriatr Psychiatry 11: 46-52.

American Psychiatric Association. 2014. Diagnostic and Statistical Manual of Mental Disorders. American Psychiatric Association: Washington, DC.

Baudic S, Tzortzis C, Barba GD, Traykov L. 2004. Executive deficits in elderly patients with major unipolar depression. J Geriatr Psychiatry Neurol 17: 195-201.

Benedict RH. 1997. Brief Visuospatial Memory Test-revised Professional Manual. Psychological Assessment Resources, Inc.: Lutz, FL.

Benton AL, Hamsher K, Sivan AB. 1994. Multilingual Aphasia Examination. AJA Associates: Iowa City, IA.

Blin O, Ferrandez AM, Serratrice G. 1990. Quantitative analysis of gait in Parkinson patients: increased variability of stride length. J Neurol Sci 98: 91-97.

Brown LA, Gage WH, Polych MA, Sleik RJ, Winder TR. 2002. Central set influences on gait. Age-dependent effects of postural threat. Exp Brain Res 145: 286-296.

Butters MA, Whyte EM, Nebes RD, et al. 2004. The nature and determinants of neuropsychological functioning in late-life depression. Arch Gen Psychiatry 61: 587-595.

Chang JT, Morton SC, Rubenstein LZ, et al. 2004. Interventions for the prevention of falls in older adults: systematic review and meta-analysis of randomized clinical trials. BMJ 328: 680-687.

Charlson ME, Pompei P, Ales KL, Mackenzie CR. 1987. A new method for classifying prognostic comorbidity in longitudinal studies: development and validation. $J$ Chronic Dis 40: 373-383.

Delis DC, Kramer JH, Kaplan E, Ober BA. 2000. California Verbal Learning Test Second Edition Manual. The Psychological Corporation: USA.

Field A. 2009. Non-parametric tests. In Discovering Statistics Using SPSS. SAGE: London; 539-583.

Folstein MF, Folstein SE, McHugh PR. 1975. "Mini-mental state". A practical method for grading the cognitive state of patients for the clinician. J Psychiatr Res 12: $189-198$.

Hajjar I, Yang F, Sorond F, et al. 2009. A novel aging phenotype of slow gait, impaired executive function, and depressive symptoms: relationship to blood pressure and other cardiovascular risks. J Gerontol A Biol Sci Med Sci 64A: 994-1001. DOI:10.1093/gerona/glp075.

Hamilton M. 1960. A rating scale for depression. J Neurol Neurosurg Psychiatry 23: 56-62.

Hartikainen S, Lonnrood E, Louhivouri K. 2007. Medication as a risk factor for falls: critical systemic review. J Gerontol A Biol Sci Med Sci 62A: 1172-1181.

Hausdorff JM, Edelberg HK, Mitchell SL, Goldberger AL, Wei JY. 1997. Increased gait unsteadiness in community-dwelling elderly fallers. Arch Phys Med Rehabil 78: 278-283.

Hausdorff JM, Rios DA, Edelberg HK. 2001. Gait variability and fall risk in community-living older adults: a 1-year prospective study. Arch Phys Med Rehabil 82: $1050-1056$.

Herman T, Giladi N, Gurevich T, Hausdorff JM. 2005. Gait instability and fractal dynamics of older adults with "cautious" gait: why do certain adults walk fearfully? Gait Posture 21: 178-185.

Herman T, Mirelman A, Giladi N, Schweiger A, Hausdorff JM. 2010. Executive control deficits as a prodrome to falls in healthy older adults: a prospective study 
linking thinking, walking, and falling. J Gerontol A Biol Sci Med Sci 65A: 1086-1092.

Holtzer R, Mahoney J, Verghese J. 2014. Intraindividual variability in executive functions but not speed of processing or conflict resolution predicts performance differences in gait speed in older adults. J Gerontol A Biol Sci Med Sci 69: 980-986. DOI:10.1093/gerona/glt180.

Hsu YH, Liang CK, Chou MY, et al. 2014. Association of cognitive impairment, depressive symptoms and sarcopenia among healthy older men in the veterans retirement community in southern Taiwan: a cross-sectional study. Geriatr Gerontol Intand 14: 102-108. DOI:10.1111/ggi.12221.

Iaboni A, Flint AJ. 2013. The complex interplay of depression and falls in older adults. Am J Geriatr Psychiatry 21: 484-492.

Lenehan ME, Summers MJ, Saunders NL, Summers JJ, Vickers JC. 2015. Relationship between education and age-related cognitive decline: a review of recent research. Psychogeriatrics . DOI:10.1111/psyg.12083.

Lesser IM, Boone KB, Mehringer M, et al. 1996. Cognition and white matter hyperintensities in older depressed patients. Am J Psychiatry 153: 1280-1286.

Lezak M, Howieson DB, Bigler ED, Tranel D. 2013. Neuropsychological Assessment. 5th edn.Oxford University Press: New York.

Lockwood KA, Alexopoulos GS, van Gorp WG. 2002. Executive dysfunction in geriatric depression. Am J Psychiatry 159: 1119-1126.

Lord S, Galna B, Verghese J, et al. 2013. Independent domains of gait in older adults and associated motor and nonmotor attributes: validation of a factor analysis approach. J Gerontol A Biol Sci Med Sci 68: 820-827.

Martin KL, Blizzard L, Wood AG, et al. 2013. Cognitive function, gait, and gait variability in older people: a population-based study. J Gerontol A Biol Sci Med Sci 68: 726-732.

Miyake A, Friedman NP, Emerson MJ, et al. 2000. The unity and diversity of executive functions and their contributions to complex "frontal lobe" tasks: a latent variable analysis. Cognitive Psychol 41: 49-100. DOI:10.1006/cogp.1999.0734.

Nakamura T, Meguro K, Sasaki H. 1996. Relationship between falls and stride length variability in senile dementia of the Alzheimer type. Gerontology 42: $108-113$.
Rapport LJ, Hanks RA, Millis SR, Deshpande SA. 1998. Executive functioning and predictors of falls in the rehabilitation setting. Arch Phys Med Rehabil 79: 629-633. Reitan R, Reitan WD. 1985. The Halstead-Reitan Neuropsychological Test Battery. Neuropsychology Press: Tuscon, AZ.

Sheridan PL, Solomont J, Kowall N, Hausdorff JM. 2003. Influence of executive function on locomotor function: divided attention increases gait variability in Alzheimer's disease. J Am Geriatr Soc 51: 1633-1637.

Spitzer RL, William JB, Gibbon M, First MB. 1994. Structured Clinical Interview for DSM-IV (SCID-IV). American Psychiatric Press: Washington D.C.

Springer S, Giladi N, Peretz C, et al. 2006. Dual-tasking effects on gait variability: the role of aging, falls, and executive function. Mov Disord 21: 950-957.

Strauss E, Sherman E, Spreen O. 2006. A Compendium of Neuropsychological Tests. Oxford University Press: Oxford, UK.

Taylor ME, Delbaere K, Lord SR, et al. 2014. Neuropsychological, physical, and functional mobility measures associated with falls in cognitively impaired older adults. $J$ Gerontol A Biol Sci Med Sci 69: 987-995. DOI:10.1093/gerona/glt166.

Vellas BJ, Wayne SJ, Romero LJ, Baumgartner RN, Garry PJ. 1997. Fear of falling and restriction of mobility in elderly fallers. Age Ageing 26: 189-193.

Wright SL, Kay RE, Avery ET, Giordani B, Alexander NB. 2011. The impact of depression on dual tasking among patients with high fall risk. J Geriatr Psychiatry Neurol 24: 142-150.

Yogev G, Hausdorff JM, Giladi N. 2008. The role of executive function and attention in gait. Mov Disord 23: 329-342.

\section{Supporting information}

Additional supporting information may be found in the online version of this article at the publisher's website. 